\title{
Fatores de risco relacionados às desordens temporomandibulares em atletas - revisão da literatura
}

\author{
Risk factors related to temporomandibulars disorders \\ In athletes - a literature review
}

\author{
Alexander Costa Moraes Silva ${ }^{1}$ \\ ANDRÉ MATHIAS CASTELLAIN SIMÕES ${ }^{1}$ \\ GENÂINE JOSÉ MARIA ${ }^{1}$ \\ SUZI MOREIRA MARTINS ${ }^{1}$ \\ BEATRIZ NETTO HAUCK ${ }^{2}$ \\ VALQUÍRIA QUINELATO ${ }^{3}$ \\ RAFAELA LADEIRA BONATO ${ }^{4}$ \\ LETÍCIA LADEIRA BONATO ${ }^{4}$
}

\section{RESUMO}

Para competir nos diversos níveis, é imprescindível que os atletas estejam saudáveis, sendo a saúde bucal parte integrante da saúde geral e do bem-estar. Contudo, durante a prática esportiva há a exposição de diversos fatores de risco, que podem se relacionar com o desenvolvimento de dor orofacial e variados tipos de desordens temporomandibulares (DTM). OBJETIVO: Revisar a literatura sobre os possíveis fatores de risco envolvidos no desenvolvimento das DTM em atletas, assim como apresentar a prevalência dessas desordens nas diversas modalidades esportivas. MÉTODOS: As bases de dados consultadas foram PubMed, BVS e Scielo por meio dos descritores "temporomandibular disorder", "athletes" e "sport". A seleção foi realizada no período de 2000 a 2020. Os resultados dos estudos mostram que macrotraumas; aumento na tolerância à dor; estresse psicológico; realização de hábitos parafuncionais; sobrecarga músculo articular devido a treinos constantes; alterações hormonais e genéticas podem aumentar as chances de atletas desenvolverem DTM, sendo que a prevalência dessa desordem varia de acordo com a modalidade esportiva, assim como sexo e idade do atleta. A presença da sintomatologia dolorosa causada pelas DTM impacta não apenas no desempenho, mas principalmente na qualidade de vida do indivíduo. Quando a dor se cronifica, pode haver a associação com a presença de dor em outras articulações do corpo, relacionadas aos mecanismos de sensibilização periférica e central. CONCLUSÃO: Atletas acometidos por DTM passam a apresentar baixa resistência a tarefas motoras que envolvem demanda e resistência. Uma abordagem interdisciplinar deve ser estimulada, de forma que o manejo e a orientação destes pacientes sejam feitos precocemente, evitando a piora na qualidade de vida e comprometimento em atividades esportivas.

Palavras-chave: Dor orofacial. Transtornos da Articulação Temporomandibular. Traumatismos em Atletas. Desempenho Atlético.

\begin{abstract}
To compete at different levels, it is essential that athletes are healthy, with oral health being an integral part of general health and well-being. However, during sports practice there is exposure of several risk factors, which can be related to the development of orofacial pain and various types of temporomandibular disorders (TMD). OBJECTIVE: To review the literature on the possible risk factors involved in the development of TMD in athletes, as well as to present the prevalence of these disorders in different sports. METHODS: The databases consulted were PubMed, BVS and Scielo through the descriptors "temporomandibular disorder", "athletes" and "sport". The selection was carried out in the period from 2000 to 2020. The results
\end{abstract}

Graduando (a) da Faculdade de Odontologia do Centro Universitário Estácio Juiz de Fora, Juiz de Fora - MG, Brasil.

Graduação em Odontologia; Especialização em andamento em Dor Orofacial e Desordem Temporomandibular, Faculdade Arthur Sá Earp Neto, Petrópolis - RJ, Brasil.

3 PhD em Odontologia, Universidade Federal Fluminense, Niterói - RJ, Brasil.

4 Professora da Faculdade de Odontologia do Centro Universitário Estácio Juiz de Fora, Juiz de Fora - MG, Brasil. 
of the studies show that macrotrauma; increased pain tolerance; psychological stress; performance of parafunctional habits; articular muscle overload due to constant training; hormonal and genetic changes can increase the chances of athletes developing TMD, and the prevalence of this disorder varies according to the sport, as well as the sex and age of the athlete. The presence of painful symptoms caused by TMD has an impact not only on performance, but mainly on the individual's quality of life. When the pain is chronic, there may be an association with the presence of pain in other joints of the body, related to the peripheral and central sensitization mechanisms. CONCLUSION: Athletes affected by TMD have low resistance to motor tasks that involve demand and resistance. An interdisciplinary approach should be encouraged, so that the management and guidance of these patients is done early, avoiding the worsening of quality of life and impairment in sports activities.

Keywords: Facial Pain; Temporomandibular Joint Disorders; Athletic Injuries; Athletic Performance.

\section{INTRODUÇÃO}

As desordens temporomandibulares (DTM) são caracterizadas por um conjunto de condições dolorosas e/ou não funcionais envolvendo a musculatura mastigatória e/ ou as articulações temporomandibulares ${ }^{1}$. Os quadros dolorosos associados a essas desordens podem afetar as atividades diárias dos indivíduos, o funcionamento psicossocial e a qualidade de vida. ${ }^{2}$

Entre os fatores etiológicos, podem ser citados dois modelos contrastantes, porém complementares. Em um modelo, os sintomas clínicos são consequências da desregulação em sistemas além dos tecidos mastigatórios, como o sistema nervoso central. Desta forma, a dorno sistema mastigatório é uma manifestação primária, e movimentos limitados da mandíbula e problemas na ATM são consequências. ${ }^{3} \mathrm{Em}$ outro modelo, comportamentos parafuncionais orais crônicos ou macrotraumas podem causar danos aos tecidos mastigatórios; alterações nociceptivas periféricas passam a ser uma consequência desse dano, contribuindo para a dor e limitação funcional. Uma possibilidade é que ocorram efeitos bidirecionais entre danos nos tecidos mastigatórios e sistemas regulatórios da dor. Além disso, acredita-se que um modelo explique o desenvolvimento inicial das DTM, enquanto o outro relaciona-se com a cronicidade das mesmas..$^{2,3}$

Neste contexto, constituindo fatores de risco, as práticas esportivas são responsáveis por muitas lesões traumáticas e não traumáticas, ${ }^{4}$ estando os atletas expostos a fatores que se inter-relacionam com as DTM, como: macrotraumas; ${ }^{4}$ aumento na tolerância à dor quando comparados com indivíduos normalmente ativos; ${ }^{5}$ estresse psicológico; ${ }^{6}$ realização de hábitos parafuncionais, como o bruxismo $;^{7}$ sobrecarga músculo articular devido a treinos constantes; alterações hormonais ${ }^{8} \mathrm{e}$ genéticas. ${ }^{9}$

As DTM frequentemente trazem limitações físicas e piora na qualidade de vida. Essas condições dificultam a rotina de um atleta de alto rendimento. Desta forma, é possível que o atleta com dor e/ou disfunção por DTM tenha queda de rendimento e performance, além de comprometimento da qualidade de vida de forma geral. ${ }^{10}$

Diante do exposto, devido à complexidade das condições e a variedade de distúrbios associados às práticas esportivas e a presença das DTM, ressalta-se a necessidade de um conhecimento detalhado sobre possíveis fatores individuais e coletivos a fim de direcionar condutas específicas para a prevenção e tratamento de tais desordens em atletas. Nesta enseada, o presente estudo busca elucidar essa relação por meio de uma revisão da literatura.

\section{MATERIAL E MÉTODOS}

Trata-se de um estudo de revisão narrativa, a qual não utiliza critérios sistemáticos para a busca e análise 
crítica da literatura. A abordagem metodológica permitiu a construção de uma contextualização da problemática e a análise das possibilidades presentes na literatura consultada para a concepção do referencial teórico da pesquisa. A seleção de artigos foi realizada no banco de dados PubMed (US National Library of Medicine), Portal da Biblioteca Virtual em Saúde (BVS) e Scielo por meio dos descritores "temporomandibular disorder", "athletes" AND "sport". A busca foi realizada em março e abril de 2020.
Os critérios de inclusão foram todos os estudos que apresentaram informações sobre dores orofaciais em atletas, com ênfase nas desordens temporomandibulares, e também aqueles que pudessem elucidar o mecanismo fisiopatológico envolvido nas DTMs (Fluxograma 1). Foram excluídos do estudo artigos duplicados e inadequados ao objetivo proposto, como abordagens de outras desordens sistêmicas e/ou que não se relacionassem ao grupo de atletas.

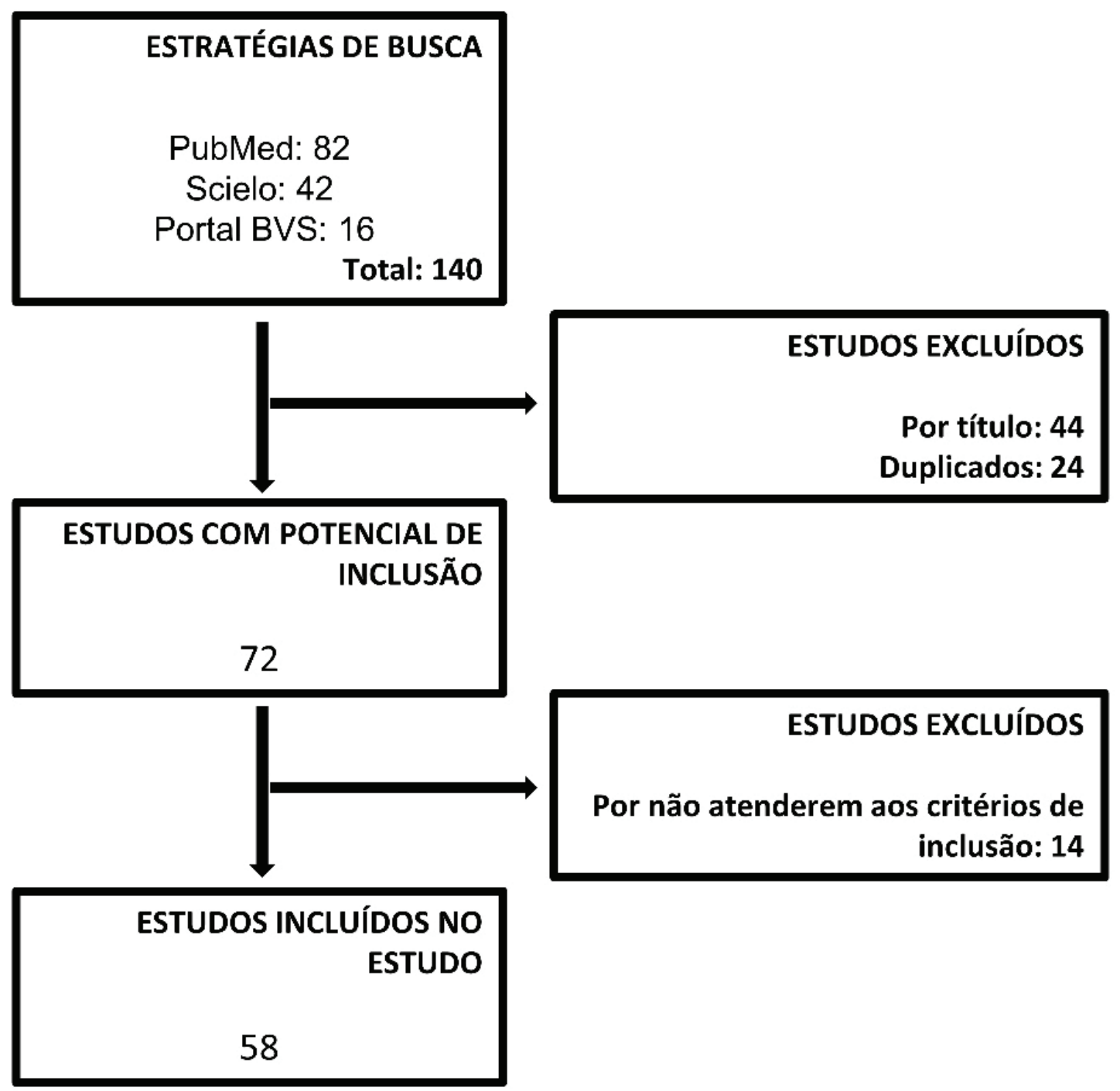

Fluxograma 1 - Seleção e inclusão dos artigos no estudo. 


\section{REVISÃo DA LITERATURA}

\section{Características da saúde bucal dos atletas}

Para competir nos diversos níveis, os atletas precisam estar bem preparados, em boa forma e saudáveis, e a saúde bucal é parte integrante da saúde geral e do bem-estar. ${ }^{11} \mathrm{O}$ comprometimento da saúde bucal pode acarretar consequências locais e também induzir respostas inflamatórias sistêmicas. ${ }^{12}$

Porém, em contraste com a percepção comum de que os atletas são "sistemicamente saudáveis”, a saúde bucal dos mesmos é considerada ruim. A cárie está presente em cerca de $75 \%$ dos atletas de elite, e outros problemas bucais, como doença periodontal, erosão dentária e traumatismos dentários também possuem alta prevalência, sendo que o comprometimento da saúde bucal tem efeito negativo no treinamento e desempenho, de acordo com relatos dos próprios atletas. ${ }^{13}$

O padrão alimentar projetado para atender às altas demandas de energia dos atletas pode coincidir com um alto risco de desenvolver cárie dentária e erosão. ${ }^{14}$ As altas demandas de energia são frequentemente atendidas pela ingestão frequente de alimentos que contêm carboidratos fermentáveis e bebidas esportivas ácidas. Sessões longas de treinamento podem resultar em diminuição do fluxo salivar devido à desidratação e respiração bucal. ${ }^{15}$

Atletas olímpicos em treinamento têm acesso a cuidados médicos por meio do Comitê Olímpico Nacional, com pouco ou nenhum custo, o que é importante, pois muitos atletas possuem recursos financeiros limitados. Porém, poucos Comitês Olímpicos Nacionais têm programas para oferecer atendimento odontológico a seus atletas. Desta forma, durante as olimpíadas, é possível observar que muitos atletas procuram atendimento odontológico eletivo, pois esses serviços são fornecidos gratuitamente. Porém, durante o período em que estão treinando, negligenciam os cuidados com a saúde bucal. ${ }^{16}$

\section{Traumas faciais}

Todas as atividades esportivas têm um risco associado de lesões orofaciais devido a quedas e colisões com jogadores, dispositivos e/ou superfícies duras. ${ }^{17}$ Os traumas esportivos representam seis vezes mais lesões faciais em comparação aos acidentes de trabalho e são responsáveis por três vezes mais lesões do que violência ou acidentes de trânsito. ${ }^{18}$ Muitas das lesões orofaciais curam sem nenhum sintoma subjetivo, mas as consequências frequentemente permanecem. ${ }^{17} \quad$ Esses traumatismos apresentam, porém, uma particularidade frente a outros: a possibilidade de prevenção, diminuindo ou eliminando a ocorrência e a gravidade das lesões nas estruturas faciais. ${ }^{19}$

Elhammali et al., $2010^{20}$ avaliaram os dados de 3596 pacientes hospitalizados por fratura maxilofacial ou fraturas da base do crânio durante um período de seis anos; 147 (4\%) desses casos foram relacionados ao esporte. As fraturas resultaram principalmente de esportes com bola (74\%), principalmente futebol $(59 \%)$, seguidos de handebol $(8 \%)$ e basquete $(1,4 \%)$. Em consequência da intensidade e duração dos treinamentos necessários para competições de elite, atletas de alto rendimento podem apresentar de duas a cinco vezes mais lesões traumáticas que atletas de recreação. ${ }^{21}$

Grande parte dos problemas da articulação temporomandibular (ATM) são causados por trauma. As injúrias das ATM relacionadas aos esportes não são imediatamente aparentes, uma vez que a maioria dos traumas mandibulares não resulta em fraturas. Forças significativas transmitidas aos tecidos moles das ATM e estruturas de suporte podem resultar em distúrbios severos. ${ }^{22,23}$ As injúrias às ATM parecem o segundo tipo de lesão mais comum 
em atividades esportivas (13,4\%), atrás apenas das lacerações e contusões de tecidos moles. ${ }^{24}$

Decorrente do trauma, forças significativas são transmitidas ao disco articular e estruturas de suporte podem resultar em ferimentos graves. O côndilo pode ser forçado posteriormente a comprimir os tecidos retrodiscais..$^{25} \mathrm{Em}$ alguns casos, o trauma das ATM pode causar hemorragia intracapsular, levando à anquilose da articulação. ${ }^{22}$

\section{Percepção dolorosa em atletas}

A percepção da dor é o resultado de mecanismos inibitórios e facilitadores concorrentes que trabalham em circuitos moduladores endógenos. ${ }^{26}$ É reconhecido que há redução imediata, embora temporária, da sensibilidade à dor após o exercício físico. Por outro lado, os efeitos a longo prazo da atividade física regular na percepção da dor não são claros. ${ }^{27}$

Expostos frequentemente a experiências sensoriais desagradáveis durante seus esforços físicos diários, os atletas são forçados a desenvolver habilidades eficientes para lidar com a dor, devido à sua exposição sistemática a breves períodos de dor intensa. ${ }^{5}$ Desta forma, acredita-se que os fatores psicológicos são responsáveis por desempenharem um papel importante no aumento da tolerância à dor em atletas, ${ }^{5}$ uma vez que muitos acreditam na teoria de "no pain, no gain." ${ }^{28} \mathrm{Em}$ uma revisão sistemática da literatura, Tesarz et al., 2012, ${ }^{5}$, apontaram que os atletas possuem maior tolerância à dor em comparação aos controles normalmente ativos; porém apresentam similar limiar doloroso, enfatizando mais uma vez o papel dos aspectos psicológicos. Desta forma, deve-se considerar que o aumento da tolerância dolorosa de atletas pode predispor a um risco aumentado de lesões neste grupo. ${ }^{27}$

As desordens temporomandibulares são a condição crônica orofacial mais comum encontrada de forma geral na população, ${ }^{29}$ assim como em atletas de alto nível, em que a participação esportiva pode causar quadros de dores crônicas. ${ }^{30,31}$ WEILER et al., 2010,31 compararam adolescentes atletas e não atletas quanto à presença de sinais e sintomas de DTM, não encontrando diferenças estatisticamente significativas. Em relação aos meninos, o sintoma com maior prevalência foi a dor muscular mastigatória à palpação (66,8\% em atletas e $60 \%$ em não atletas), acompanhado de aperto ou ranger de dentes em atletas (50\%) e estalidos em não atletas $(20 \%)$. Em relação ao grupo das meninas atletas, os sinais e sintomas mais prevalentes de DTM foram de dor muscular mastigatória à palpação, desvio de abertura/fechamento e apertamento e/ou trituração dos dentes. Já nos grupos das não atletas, os sinais e sintomas foram de dor muscular à palpação, seguida de desvio de abertura / fechamento e dor durante a abertura.

\section{Realização do hábito parafuncional do bruxismo}

O bruxismo é definido como a atividade muscular mastigatória repetitiva caracterizada por apertar, ranger, encostar os dentes ou movimentar a mandíbula em períodos não funcionais, sendo um dos fatores de risco para o desenvolvimento de dor muscular e nas ATM. ${ }^{32}$

Pesquisas sugerem que 0 estresse pode ser um dos fatores de risco envolvidos no desenvolvimento do bruxismo em cerca de $70 \%$ dos indivíduos, ${ }^{33}$ Além de aspectos psicológicos, atletas de alto desempenho desenvolvem um aumento da massa muscular, que também inclui os músculos mastigatórios. Esses músculos tendem a se contrair com mais frequência e com maior intensidade, o que leva a forças oclusais de amplitude excessiva. ${ }^{7}$

Uma das causas atribuídas à hipertrofia muscular é de que, ao tentar suportar a dor durante a prática esportiva, percebe-se 
frequentemente que os atletas realizam 0 apertamento dos dentes. A quantidade de horas de treinamento e o número de anos de prática de esportes de alta performance estão fortemente correlacionados com o dano dentário. ${ }^{7}$ WEILER et al., 2010,31, estudando adolescentes do sexo masculino, observaram que $50 \%$ dos atletas realizavam bruxismo, e nenhum dos meninos não atletas realizava o hábito parafuncional.

\section{Hormônios}

A alta prevalência de DTM dolorosas em mulheres, o padrão de início após a puberdadee as taxas de prevalência mais baixas no período pós-menopausa sugerem que os hormônios reprodutivos femininos desempenham umpapel etiológico fundamental no desenvolvimento das desordens articulares, ${ }^{34,35}$ como também demonstrado por estudos anteriores do nosso grupo de pesquisa. ${ }^{36}$

A incidência de lesões esportivas é maior em atletas do sexo feminino quando comparadas aos do sexo masculino. ${ }^{37} \mathrm{~A}$ prática de exercícios extenuantes tem sido associada com vários distúrbios do ciclo menstrual, incluindo retardo puberal, defeitos na fase lútea, anovulação e amenorreia. ${ }^{38}$ O treinamento físico intenso pode alterar a liberação de gonadotrofinas e, dessa forma, induzir alterações do ciclo menstrual. Acreditase que se trata de um mecanismo adaptativo para poupar energia e, assim, proteger importantes processos fisiológicos. Contudo, hormônios sexuais possuem importantes funções fisiológicas, sendo que alterações podem causar prejuízos. ${ }^{39}$

Coelho et al., 2015, ${ }^{39}$ observara que o comportamento dos hormônios de atletas de elite de ginástica rítmica em uma fase précompetição apresentou padrão diferente do esperado para mulheres de mesma idade e com ciclo menstrual regular. Contribuindo para as disfunções hormonais, cita-se ainda o treinamento físico intenso, o estresse psicológico e a escassez energética, considerados também fatores de risco para o desenvolvimento das DTM. ${ }^{7,33}$

\section{Alterações genéticas}

De forma geral, as dores musculares esqueléticas, incluindo as mastigatórias, possuem uma complexa fisiopatologia, envolvendo fenômenos periféricos e centrais que podem, além de outras formas, serem induzidos por alterações genéticas relacionadas aos processos de formação e regeneração muscular. ${ }^{40}$

$\mathrm{Em}$ relação às tendinopatias, apesar do conhecimento da influência de fatores extrínsecos, a tolerância à carga de cada atleta é única. Há ampla variação interindividual na resposta a processos musculoesqueléticos. Desta forma, também observado pelo nosso grupo de pesquisa em estudo prévio, alterações genéticas podem predispor atletas ao desenvolvimento de tendinopatias. ${ }^{9}$ Alterações genéticas também podem estar associadas à presença de desordens temporomandibulares dolorosas, assim como associadas à presença de dores crônicas em outras partes do corpo.. ${ }^{41}$

$\mathrm{Na}$ tabela 1 estão demonstrados estudos que correlacionaram a presença de desordens temporomandibulares com a prática esportiva.

\section{DIscussão}

As DTM dolorosas são ao mesmo tempo consequências e causas de alterações em fatores clínicos, psicossociais e biológicos. Desta forma, torna-se árduo demonstrar o impacto que um único fator de risco tem no aparecimento de sinais e sintomas desta desordem. ${ }^{25}$ Contudo, sabe-se que atletas estão claramente mais expostos a fatores de riscos comuns ao desenvolvimento das DTM, ${ }^{24,25}$ como traumas faciais ${ }^{25}$, estresse e ansiedade. ${ }^{7}$ Assim sendo, a observação e orientação desses indivíduos torna-se extremamente necessária. ${ }^{25}$ 
FATORES DE RISCO RELACIONADOS ÀS DESORDENS

TEMPOROMANDIBULARES EM ATLETAS - REVISÃO DA LITERATURA

Tabela 1 - Estudos correlacionando sinais e sintomas de DTM em atletas.

\begin{tabular}{|c|c|c|c|c|c|}
\hline AUTOR & ANO & AMOSTRA & POPULAÇÃO & ESPORTE & RESULTADO \\
\hline $\begin{array}{c}\text { Tozoglu, S \& Tozoglu, } \\
\qquad U^{42}\end{array}$ & 2006 & 64 & Turquia & Futebol amador & $\begin{array}{l}\text { Foram observadas fraturas dento- } \\
\text { alveolares }(36 \%) \text {, distúrbios da articulação } \\
\text { temporomandibular }(27 \%) \text {, fraturas } \\
\text { mandibulares }(27 \%) \text { e fraturas nasais }(9 \%) \text {. }\end{array}$ \\
\hline Jagger et al. ${ }^{43}$ & 2009 & 200 & Austrália & Mergulho & $\begin{array}{l}\text { A prevalência de dor orofacial relatada foi } \\
\text { de } 44 \% .21 \% \text { relataram dor de dente, } 27 \% \\
\text { dor sinusal, } 16 \% \text { de dor nas ATM e } 12 \% \\
\text { outras dores. }\end{array}$ \\
\hline Gay Escoda et al. ${ }^{44}$ & 2011 & 30 & Barcelona & $\begin{array}{l}\text { Futebol } \\
\text { profissional }\end{array}$ & $\begin{array}{l}30 \% \text { dos atletas avaliados apresentaram } \\
\text { bruxismo; } 16,7 \% \text { estalidos articulares e } \\
6,7 \% \text { reportaram dor à palpação articular. }\end{array}$ \\
\hline Lešić et al. ${ }^{24}$ & 2011 & 195 & Croácia & Basquete & $\begin{array}{l}\text { Foram observadas } 2615 \text { lesões no } \\
\text { sistema estomatognático. As lesões mais } \\
\text { comuns foram lacerações e contusões } \\
\text { de tecidos moles }(84,4 \%) \text {, lesões da } \\
\text { articulação temporomandibular e rigidez } \\
\text { dos músculos orais }(13,4 \%) \text { e lesões } \\
\text { dentárias }(2,2 \%) \text {. }\end{array}$ \\
\hline Lobbezzo et al. ${ }^{45}$ & 2014 & 536 & Holanda & Mergulho & $\begin{array}{l}\text { As queixas de dor por DTM foram } \\
\text { relatadas por } 44,1 \% \text { dos mergulhadores. } \\
\text { O apertamento dental contribuiu para as } \\
\text { dores faciais, assim como o mergulho em } \\
\text { água fria atuou como fator de proteção } \\
\text { para a dor associadas às DTM. }\end{array}$ \\
\hline $\begin{array}{l}\text { Mendoza-Puente et } \\
\text { al. }^{46}\end{array}$ & 2014 & 38 & Espanha & $\begin{array}{l}\text { Boxe e } \\
\text { handebol }\end{array}$ & $\begin{array}{l}\text { Os boxeadores profissionais do sexo } \\
\text { masculino apresentaram maior impacto } \\
\text { da dor de cabeça e sensibilização local da } \\
\text { região craniomandibular do que os jogadores } \\
\text { profissionais de handebol. Não foi possível } \\
\text { determinar se esses achados são de curta } \\
\text { duração, como resultado da atividade de } \\
\text { treinamento, ou de longa duração. }\end{array}$ \\
\hline Bonotto et. al ${ }^{10}$ & 2016 & 82 & Brasil & Artes marciais & $\begin{array}{l}\text { Os praticantes de artes marciais } \\
\text { apresentaram mais diagnósticos de DTM } \\
\text { (média de } 45,6 \% \text { ) do que os não atletas } \\
(14,3 \%) \text {. Os diagnósticos de deslocamento } \\
\text { de disco e a dor miofascial foram as DTM } \\
\text { mais comuns entre os atletas. }\end{array}$ \\
\hline $\begin{array}{l}\text { Duplat, IS \& Nunes, } \\
\qquad \mathrm{AM}^{47}\end{array}$ & 2018 & 51 & Brasil & Boxe & $\begin{array}{l}\text { Dos atletas que usaram protetores bucais, } \\
20 \text { não desenvolveram a síndrome e } \\
18 \text { desenvolveram, correspondendo } \\
\text { a } 52,6 \% \text { e } 47,4 \% \text {, respectivamente. } \\
\text { Houve associação entre participação } \\
\text { em campeonatos e presença de DTM, } \\
\text { demonstrando que atletas de competição } \\
\text { apresentam grande fator de risco para } \\
\text { desenvolvimento da disfunção. }\end{array}$ \\
\hline Bonotto et. al ${ }^{23}$ & 2019 & 58 & Brasil & Rugby & $\begin{array}{l}\text { Os jogadores de rugby apresentaram } \\
\text { prevalência de DTM de } 53,3 \% \text { e o } \\
\text { grupo controle de } 14,3 \%(p=0,002) \text {. } \\
\text { Dentre os jogadores, a dor miofascial foi } \\
\text { observada em } 40 \%, 23,3 \% \text { apresentaram } \\
\text { deslocamento de disco e os atletas } \\
\text { também relataram apertamento dentário } \\
\text { significativamente maior do que os do } \\
\text { grupo controle }(p=0,003) \text {. }\end{array}$ \\
\hline
\end{tabular}


A presença da sintomatologia dolorosa causada pelas DTM impacta não apenas no desempenho, mas principalmente na qualidade de vida do indivíduo, uma vez que tanto a dor física, como a psicossomática podem propiciar limitações funcionais. ${ }^{48}$ Indivíduos acometidos por DTM passam a apresentar baixa resistência a tarefas motoras que envolvem demanda e resistência. ${ }^{49}$ Sob outro ponto de vista, e representando um importante problema de saúde pública, cerca de $10 \%$ das DTM evoluem de maneira não satisfatória, com prognóstico desfavorável, associadas à cronificação e disseminação da dor para áreas circunvizinhas. ${ }^{49,50}$ WÄNMAN, 2012 ${ }^{49}$, em seu estudo, destacou que indivíduos com DTM apresentavam frequentemente dores nas regiões cervical, de ombro e lombar. A partir de ensaio clínico prévio, nosso grupo de pesquisa também pode observar que indivíduos com DTM apresentaram alta prevalência de dor em outras articulações do corpo quando comparados com indivíduos sem a desordem, sendo que a dor no joelho é queixa comum mais prevalente, ${ }^{51}$ fatos estes que podem comprometer o desempenho nas práticas esportivas.

O tipo de lesão ou disfunção resultante depende do mecanismo de contato, frequência e forças recebidas. Desta forma, as DTM associadas às práticas esportivas podem ser provocadas por efeito direto na região das ATM e/ou regiões da mandíbula e mento, e de forma indireta, associadas a outros fatores de risco, como o hábito de apertar os dentes (bruxismo cêntrico). ${ }^{52} \mathrm{De}$ forma direta (traumas), os adolescentes são os responsáveis pelo maior número de lesões, devido principalmente ao aumento da participação em esportes coletivos nessa faixa etária, sendo que devido aos mesmos motivos, há maior prevalência de lesões em meninos do que meninas. ${ }^{42,49,53} \mathrm{~A}$ maioria dos traumas na mandíbula não resulta em fraturas, ${ }^{49}$ porém, quando estas ocorrem, acometem mais as regiões subcondilar, seguida pelo ângulo e pelo corpo da mandíbula. ${ }^{42}$ Dentre os esportes de contato, mesmo a incidência de fraturas relacionadas ao futebol não sendo as maiores, a gravidade e a extensão da lesão podem ser mais importante do que outros esportes de contato devido a impactos violentos entre jogadores, como cotovelo-cabeça e impacto frente a frente, que geralmente acontece quando a bola é jogada com a testa. ${ }^{54}$

Em relação aos fatores indiretos, sabe-se que os hormônios possuem papel importante nas DTM. ${ }^{36}$ Apesar de envolver distintos mecanismos, acredita-se que a influência ocorra diretamente sobre o metabolismo e homeostase da articulação temporomandibular $(\mathrm{ATM})^{35}$, e também na modulação dolorosa, através de sua atuação sobre o sistema nervoso central e periférico. ${ }^{55} \mathrm{Em}$ um estudo realizado por Lima et al. (2001), ${ }^{56}$ foi encontrado um nível significativamente mais alto de testosterona em atletas adolescentes do sexo masculino envolvidos em esportes de alto impacto (ginástica, atletismo e basquete) do que no grupo de esportes de baixo impacto (natação e polo aquático). Esse aumento da testosterona esteve acompanhado do aumento da densidade óssea mineral. ${ }^{56}$ Esses dados são corroborados pelo estudo de FLAKE et al., $2006^{57}$, que demonstraram que a testosterona pode atenuar e o estrogênio exacerbar os danos na ATM. Desta forma, acredita-se que esta também pode ser umas das teorias que explicam a maior prevalência de DTM em mulheres atletas do que homens.

O uso de protetores bucais é encorajado em diversos esportes, principalmente aqueles de contato. $^{25}$ Contudo, embora a frequência de fraturas dentárias diminua, Murakami et al., $2008^{58}$, encontraram diversos atletas que se queixaram de dor e DTM após o uso de protetores. Os protetores bucais são confeccionados considerando-se características dentárias. Desta forma, quando 
utilizados, pode modificar a relação oclusal, muscular e articular das ATM, alterando a posição condilar e do disco. De acordo com o estudo, os autores observaram que quando a espessura do protetor bucal é menor que 6 mm, o uso não afeta os componentes da ATM. Além disso, o uso de protetores resilientes pode estimular o apertamento dentário. ${ }^{58}$

\section{CONSIDERAÇõES finAIS}

Os atletas são grupos expostos a riscos aumentados de desenvolvimento de quadros dolorosos e disfuncionais de desordens temporomandibulares. Uma abordagem interdisciplinar deve ser estimulada, de forma que o manejo e a orientação destes pacientes, bem como, o tratamento, sejam feitos precocemente, evitando a piora na qualidade de vida e comprometimento em atividades esportivas.

Agradecimentos e conflito de interesse

Os autores declaram que não há conflitos de interesses.

Fontes de financiamento

Sem financiamento.

\section{REFERÊNCIAS}

1. Bender SD. Orofacial pain and headache: a review and look at the commonalities. Curr Pain Headache Rep. 2014;18(3):400. DOI: 10.1007/s11916-013-0400-5.

2. Schiffman E, Ohrbach R, Truelove E, Look J, Anderson G, Goulet JP, et al. Diagnostic criteria for temporomandibular disorders (DC/ TMD) for clinical and research applications: recommendations of the International $\mathrm{RDC} /$ TMD Consortium Network and Orofacial Pain Special Interest Group. J oral facial pain headache. 2014;28(1):6-27. DOI: 10.11607/ jop. 1151.
3. Ohrbach R, Bair E, Fillingim RB, Gonzalez Y, Gordon SM, Lim P, et al. Clinical Orofacial Characteristics Associated With Risk of FirstOnset TMD: The OPPERA Prospective Cohort Study. J Pain. 2013;14(12):T33-50. DOI: 10.1016/j.jpain.2013.07.018.

4. Tanaka, Y, Tsugawa, T, Maeda Y. Effect of mouthguards on impact to the craniomandibular complex. Dent Traumatol. 2017;33(1):51-56. DOI: 10.1111/edt.12283.

5. Tesarz J, Schuster AK, Hartmann M, Gerhardt A, Eich W. Pain perception in athletes compared to normally active controls : A systematic review with meta-analysis. Pain. 2012;153(6):125362. DOI: 10.1016/j.pain.2012.03.005.

6. Malm C, Jakobsson J, Isaksson A. Physical Activity and Sports-Real Health Benefits: A Review with Insight into the Public Health of Sweden. Sports (Basel). 2019 May 23;7(5):127. DOI: $10.3390 /$ sports7050127.

7. Bernhardt O, Gesch D, Schwahn C, Mack $F$, Meyer G, John U, et al. Risk factors for headache, including TMD signs and symptoms, and their impact on quality of life. Results of the Study of Health in Pomerania (SHIP). Quintessence Int. 2005 Jan;36(1):55-64.

8. Larson-Meyer DE, Gostas DE. Thyroid Function and Nutrient Status in the Athlete. Curr Sports Med Rep. 2020 Feb;19(2):84-94. DOI: 10.1249/ JSR.0000000000000689.

9. Salles JI, Amaral MV, Aguiar DP, Lira DA, Quinelato V, Bonato LL, et al. BMP4 and FGF3 haplotypes increase the risk of tendinopathy in volleyball athletes. J Sci Med Sport. 2015;18(2):150-5. DOI: 10.1016/j. jsams.2014.02.011.

10. Bonotto D, Namba EL, Veiga DM, Wandembruck $F$, Mussi F, Cunali PA, et al. Professional karatedo and mixed martial arts fighters present with a high prevalenceoftemporomandibulardisorders. Dental traumatology. 2016;32(4):281-5. DOI: 10.1111/edt.12238.

11. Gallagher J, Ashley P, Petrie A, Needleman I. Oral health and performance impacts in elite and professional athletes. Community Dent Oral Epidemiol. 2018;46(4):563-8. DOI: 10.1111/cdoe.12392. 
12. Cullinan MP, Seymour GJ. Periodontal disease and systemic illness: will the evidence ever be enough? Periodontol 2000. 2013;62(1):27186. DOI: 10.1111/prd.12007.

13. Ashley P, lorio A Di, Cole E, Tanday A, Needleman I. Oral health of elite athletes and association with performance: a systematic review. Br J Sports Med. 2015;49(1):14-9. DOI: 10.1136/bjsports-2014-093617.

14. Bryant S, Mclaughlin K, Morgaine K, Drummond B. Elite Athletes and Oral Health. Int J Sports Med. 2011;32(9):720-4. DOI: 10.1055/s-00311277192.

15. Bruins GJ, Vissink A, Veerman EC, Van Nieuw Amerongen A. Influence of sports on saliva. Nederlands Tijdschrift Voor Tandheelkunde. 2014;115(9):467-73.

16. Piccininni PM, Fasel R. Sports dentistry and the olympic games. J Calif Dent Assoc. 2005;33(6):471-83.

17. Lešić $N$, Seifert $D$, Zečić $M$, Pezo H. Differences in Movement of Temporomandibular Joints in Athletes With and Without Orofacial Injuries. Coll Antropol. 2016;40(3):189-93.

18. Tuli $T$, Hächl $O$, Hohlrieder $M$, Grubwieser G, Gassner R. Dentofacial trauma in sport accidents. Gen Dent. 2002;50(3):274-279.

19. Young EJ, Macias CR, Stephens L. Common Dental Injury Management in Athletes. Sports Health. 2015 May;7(3):250-5. DOI: 10.1177/1941738113486077.

20. Elhammali N, Bremerich A, Demographical JR. Demographical and clinical aspects of sportsrelated maxillofacial and skull base fractures in hospitalized patients. Int $\mathbf{J}$ Oral Maxillofac Surg. 2010;39(9):857-62. DOI: 10.1016/j. ijom.2010.04.006.

21. Maffulli N, Baxter-Jones AD, Grieve A. Long term sport involvement and sport injury rate in elite young athletes. Arch Dis Child. 2005;90(5):525-7. DOI: 10.1136/ adc. 2004.057653 .

22. Sharma S, Ohrbach 2, Fillingim RB, Greenspan JD, Slade G. Pain Sensitivity Modifies Risk of Injury-Related Temporomandibular Disorder. J Dent Res. 2020 May;99(5):530-536. DOI: 10.1177/0022034520913247.
23. Bonotto D, Penteado CA, Namba EL, Cunali PA, Rached RN, Azevedo-Alanis LR. Prevalence of temporomandibular disorders in rugby players. Gen Dent. 2019;67(4):72-74.

24. Lesić N, Seifert D, Jerolimov V. Orofacial injuries reported by junior and senior basketball players. Coll Antropol. 2011;35(2):347-352.

25. Weiler RM, Santos FM, Kulic MA, Lima MP, Pardini SR, Mori M, et al. Prevalence of signs and symptoms of temporomandibular dysfunction in female adolescent athletes and non-athletes. Int J Pediatr Otorhinolaryngol. 2013;77(4):519_ 524. DOI: 10.1016/j.ijporl.2012.12.024.

26. Nir RR, Yarnitsky D. Conditioned pain modulation. Curr Opin Support Palliat Care. 2015;9(2):131-137. DOI: 10.1097/ SPC. 0000000000000126.

27. Flood A, Waddington G, Thompson K, Cathcart S. Increased conditioned pain modulation in athletes. J Sports Sci. 2017;35(11):10661072. DOI: $10.1080 / 02640414.2016 .1210196$.

28. Schneider S, Sauer J, Berrsche G, Schmitt H. No Pain, No Gain? Prevalence, Location, Context, and Coping Strategies with Regard to Pain Among Young German Elite Basketball Players. J Hum Kinet. 2019 Oct 18;69:179189. DOI: 10.2478/hukin-2018-0098.

29. Rollman GB, Gillespie JM. The Role of Psychosocial Factors in Temporomandibular Disorders. Current review of pain. 2000;4(1):71-81.

30. Hainline B, Turner JA, Caneiro JP, Stewart M, Lorimer Moseley G. Pain in elite athletesneurophysiological, biomechanical and psychosocial considerations: a narrative review. Br J Sports Med. 2017;51(17):1259-1264. DOI: 10.1136/bjsports-2017-097890.

31. Weiler RME, de Souza Vitalle MS, Mori M, Kulik MA, Ide L, Pardini SRDSV, et al. Prevalence of signs and symptoms of temporomandibular dysfunction in male adolescent athletes and non-athletes. Int J Pediatr Otorhinolaryngol. 2010;74(8):896-900. DOI: 10.1016/j. ijporl.2010.05.007.

32. Lobbezoo F, Ahlberg J, Raphael KG, Wetselaar $P$, Glaros AG, Kato $T$, et al. International consensus on the assessment of bruxism: Report of a work in progress. J Oral Rehabil. 2018;45(11):837-844. DOI: 10.1111/joor.12663. 
FATORES DE RISCO RELACIONADOS ÀS DESORDENS

TEMPOROMANDIBULARES EM ATLETAS - REVISÃO DA LITERATURA

33. Shetty S, Pitti V, Babu CLS, Kumar GPS, Deepthi BC. Bruxism: A Literature Review. J Indian Prosthodont Soc. 2011;10(3):141-148. DOI: 10.1007/s13191-011-0041-5.

34. Robinson JL, Johnson PM, Kister K, Yin MT, Chen J, Wadhwa S. Estrogen signaling impacts temporomandibular joint and periodontal disease pathology. Odontology. 2020;108(2):153-165. DOI: 10.1007/s10266019-00439-1.

35. Wu Y, Kadota-Watanabe C, Ogawa T, Moriyama K. Combination of estrogen deficiency and excessive mechanical stress aggravates temporomandibular joint osteoarthritis in vivo. Arch Oral Biol. 2019;102:39-46. DOI: 10.1016/j.archoralbio.2019.03.012.

36. Quinelato V, Bonato LL, Vieira AR, Granjeiro JM, Tesch R, Casado PL. Association between polymorphismsinthegenes ofestrogenreceptors and the presence of temporomandibular disorders and chronic arthralgia. J Oral Maxillofac Surg. 2018;76(2):314. e1-314. e9. DOI: 10.1016/j.joms.2017.10.023.

37. Murphy DF, Connolly DAJ, Beynnon BD. Risk factors for lower extremity injury: a review of the literature. British journal of sports medicine. 2003;37(1):13-29.

38. Pardini DP. Alterações hormonais da mulher atleta.Arquivos Brasileiros deEndocrinologia \& Metabologia.2001;45(4):343-351.

39. Coelho SMH, Simões RD, Lunz W. Desequilibrio hormonal y la disfunción menstrual en atletas de gimnasia rítmica. Revista Brasileira de Ciências do Esporte. 2015;37(3):222-229.

40. Cairns BE. Pathophysiology of TMD painbasic mechanisms and their implications for pharmacotherapy. J Oral Rehabil. 2010;37(6):391-410. DOI: 10.1111/j.13652842.2010.02074.x.

41. Bonato LL, Quinelato V, De Felipe Cordeiro PC, De Sousa EB, Tesch R, Casado, PL. Association between temporomandibular disorders and pain in other regions of the body. J Oral Rehabil. 2017; 44(1):9-15. DOI: 10.1111/joor.12457.

42. Tozoglu S, Tozoglu U. A one-year review of craniofacial injuries in amateur soccer players. J Craniofac Surg. 2006;17(5):825-827. DOI: 10.1097/01.scs.0000234982.54121.6b.
43. Jagger RG, Shah CA, Weerapperuma ID, Jagger DC. The prevalence of orofacial pain and tooth fracture (odontocrexis) associated with SCUBA diving. Prim Dent care. 2009;16(2):75-78. DOI: $10.1308 / 135576109787909463$.

44. Gay Escoda C, Pereira DMVD, Ardèvol J, Pruna R, Fernandez J, Valmaseda Castellón E. Study of the effect of oral health on physical condition of professional soccer players of the Football Club Barcelona. Medicina Oral, Patología Oral y Cirurgia Bucal. 2011;16(3):436-439. DOI: 10.4317/medoral.16.e436.

45. Lobbezoo F, Van Wijk AJ, Klingler MC, Ruiz Vicente E, Van Dijk CJ, Eijkman MAJ. Predictors for the development of temporomandibular disorders in scuba divers. J Oral Rehabil. 2014;41(8):573-580. DOI: 10.1111/joor.12178.

46. Mendoza-Puente M, Oliva-Pascual-Vaca Á, Rodriguez-Blanco C, Heredia-Rizo AM, TorresLagares D, Ordoñez FJ. Risk of headache, temporomandibular dysfunction, and local sensitization in male professional boxers: a case-control study. Arch Phys Med Rehabil. 2014;95(10):1977-1983. DOI: 10.1016/j. apmr.2014.06.011.

47. Duplat YS, Nunes AM. Prevalência de sinais e sintomas para Disfunção Temporomandibular em lutadores de boxe. Revista Pesquisa em Fisioterapia. 2018;8(2):191-198.

48. Abe EY. Dynamic baropodometry in rowing athletes refering signs and symptoms of temporomandibular joint dysfunction before and after using an occlusal splint 11 associated to therapeutic mandibular exercise: pilot study. Rev Dor. 2016;109(1).

49. Wänman A. Endurance to physical strain in patients with temporomandibular disorders: A case-control study. Acta Odontologica Scandinavica. 2012;70(6):455-462. DOI: 10.3109/00016357.2011.635599.

50. Furquim BD, Flamengui LM, Conti PC. TMD and chronic pain: a current view. Dental Press J Orthod. 2015;127-33. DOI: 10.1590/21769451.20.1.127-133.sar.

51. Velly AM, Look JO, Carlson C, Lenton PA, Kang W, Holcroft CA, etal. The effect of catastrophizing and depression on chronic pain-a prospective cohort study of temporomandibular muscle and 
joint pain disorders. Pain. 2011;152(10):23772383. DOI: 10.1016/j.pain.2011.07.004.

52. Ginszt M, Zieliński G, Byś A, Gawda P, Majcher P. Int J Environ Res Public Health. 2020 Feb 21;17(4). DOI: 10.3390/ijerph17041378.

53. Antoun JS, Lee KH. Sports-Related Maxillofacial Fractures Over an 11-Year Period. J Oral Maxillofac Surg. 2008;66(3):504-508. DOI: 10.1016/j.joms.2007.08.018.

54. Delilbasi C, Yamazawa M, Nomura K. Maxillofacial fractures sustained during sports played with ball Maxillofacial fractures sustained during sports played with a ball. Oral Surg Oral Med Oral Pathol Oral Radiol Endod. 2004;97(1):23-27. DOI: 10.1016/j. tripleo.2003.10.008.

55. Bi R, Ding Y, Gan Y. A new hypothesis of sexdifferences in temporomandibular disorders: Estrogen enhances hyperalgesia of inflamed TMJ through modulating voltage-gated sodium channel 1.7 in trigeminal ganglion?
Med Hypotheses. 2015;84(2):100-3. DOI: 10.1016/j.mehy.2014.12.010.

56. Lima F, De Falco V, Baima J, Carazzato JG, Pereira RM. Effect of impact load and active load on bone metabolism and body composition of adolescent athletes. Med Sci Sports Exerc. 2001;33(8):1318-1323. DOI: 10.1097/00005768-200108000-00012.

57. Flake NM, Hermanstyne TO, Gold MS. Testosterone and estrogen have opposing actions on inflammation-induced plasma extravasation in the rat temporomandibular joint. Am J Physiol Regul Integr Comp Physiol. 2006;291(2):R343-R348. DOI: 10.1152/ajpregu.00835.2005.

58. Murakami S, Maeda Y, Ghanem A, Uchiyama $\mathrm{Y}$, Kreiborg S. Influence of mouthguard on temporomandibular joint. Scandinavian journal of medicine \& science in sports. 2008;18(5):591-595. DOI: 10.1111/j.16000838.2007.00698.x.

Submetido em: 16-8-2020

Aceito em: 16-10-2020 